
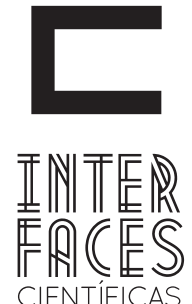

EXATAS E TECNOLÓGICAS

ISSN IMPRESSO - 2359-4934

ISSN ELETRÔNICO - 2359-4942

DOI - 10.17564/2359-4934.2015v1n2p35-46

\title{
ACADEMIC ANALYTICS: APLICANDO TÉCNICAS DE BUSINESS INTELLIGENCE SOBRE DATOS DE PERFORMANCE ACADÉMICA EN ENSEÑANZA SUPERIOR
}

Ana Magela Rodriguez Almeida ${ }^{1}$
Sandro da Silva Camargo ${ }^{2}$

\section{RESUMO}

Hoje em dia, as organizações estão armazenando e gerando um volume cada vez mais de dados. Esta situação leva a grandes desafios, como a integração e transformação de dados em informações relevantes para otimizar o processo decisório. Então, é necessário construir uma base de conhecimento para integrar e organizar os dados disponíveis, a fim de torná-los fáceis de entender e permitir o acesso rápido, permitindo a sua exploração e análise, a fim de atender às expectativas estratégicas das organizações. Nes- se cenário, este trabalho apresenta uma abordagem para a implementação de Business Intelligence em uma instituição de ensino superior, com o objetivo de melhorar significativamente a aplicação de gestão de recursos na organização.

\section{PALAVRAS-CHAVE}

Business Intelligence, Ensino Superior. Tomada De Decisão. 


\section{ABSTRACT}

Today, organizations are storing and generating an increasing volume of data. This leads to major challenges, such as integrating and transforming data into relevant information to optimize decision-making. So we need to build a knowledge base to integrate and organize the available data in order to make them easy to understand and allow quick access, allowing its exploration and analysis in order to meet the expectations of strategic organizations. In this scenario,

\section{RESUMEN}

En la actualidad, los volúmenes de datos generados y almacenados por las organizaciones crecen de forma desmesurada. Esta situación trae consigo grandes desafíos, entre ellos como integrar y transformar estos datos en informaciones relevantes de forma que se pueda optimizar el proceso de toma de decisiones en los negocios. En el contexto descripto para la efectiva utilización como apoyo al proceso de toma de decisiones, es necesaria una base para organizar e integrar los datos disponibles de fácil entendimiento y que posibilite acceder a los datos de forma rápida. Permitiendo de esta manera la exploración de datos para identificar informaciones nuevas y útiles para atender las expectativas y necesidades estratégicas de las organizaciones. El presente trabajo tiene como principal objetivo presentar un enfoque de las etapas this paper presents an approach to the implementation of Business Intelligence in a higher education institution, with the goal of significantly improving resource management application in the organization.

\section{KEYWORDS}

Business Intelligence. Higher Education. Decision-Making. de aplicación de técnicas de Business Intelligence para optimizar el proceso de toma de decisiones en una Institución de Enseñanza Superior. De esta manera con la conclusión del proyecto de investigación se pretende disponibilizar un conjunto de tecnologías y servicios que posibiliten una mejor administración de la Institución a través de análisis de informaciones relevantes y descubiertas de tendencias futuras proporcionando un entorno favorable al desenvolvimiento dinámico de las actividades de la Universidad.

\section{PALABRAS CLAVE}

Business Intelligence. Enseñanza Superior. Toma de decisiones. 


\section{INTRODUCCIÓN}

Actualmente, el período de la historia humana es conocido como Era de la Información, debido a la alta disponibilidad de los datos generados. De acuerdo con la International Data Corporation (IDC), en 2013, en el universo, el volumen de datos generados sumó 2.7 zettabytes. Otra reciente pesquisa publicada por la IDC estima que en 2020 el volumen de datos circulando por el mundo alcanzará 40zettabytes (IDC, 2012).

Los servidores de las organizaciones son un ejemplo de dominio de aplicación que genera grandes volúmenes de datos diariamente. La mayoría de las organizaciones poseen un sistema de almacenamiento de datos referentes a las actividades realizadas, de forma consolidada. Pues estos datos solamente no nos dicen mucha cosa, es necesario convertir estos datos en informaciones útiles que de alguna manera serán de mucha utilidad para las organizaciones.

El éxito de una organización, así como la ejecución de sus actividades, están centralizados en adoptar decisiones, siendo considerada una de las principales etapas en el proceso de negocios. De esta forma, la toma de decisiones estratégica en el concepto de Business Intelligence (BI) se convierte en una actividad de extrema importancia, una vez que decisiones equivocadas pueden comprometer el futuro de una organización.

De esta manera, el suceso de una organización no está solamente en la generación y almacenamiento de los volúmenes de datos referentes a sus actividades pero si, en el conocimiento de la información adquirido y en su correcta utilización. Con esto los datos proporcionados por $\mathrm{BI}$ pueden agregar valor a la toma de decisiones en las organizaciones en el sentido que este gran volumen de datos generados incita la innovación y permite la optimización del resultado del negocio.
Inseridas en el mismo contexto de las organizaciones, Instituciones de Enseñanza necesitan tomar decisiones acerca del desenvolvimiento de sus actividades. En el contexto particular de este proyecto, implementar BI en organizaciones como la Universidad Federal do Pampa (UNIPAMPA) pueden ser de alta relevancia para identificar, comprender y predecir posibles problemas residentes en la Institución, que muchas veces son resultados de decisiones tomadas sin base en el conocimiento generado por estos datos. La hipótesis de investigación de este trabajo es identificar si la aplicación de técnicas de BI puede contribuir para lo proceso de toma de decisiones en una institución de enseñanza superior.

Este artículo está organizado como sigue. En la sección dos se presentan los conceptos básicos de Business Intelligence. En la sección tres son presentados la metodología adoptada en la realización del presente trabajo y las etapas del proceso de implementación de BI en la Institución. La sección cuatro presenta los resultados obtenidos en un estudio de caso en la UNIPAMPA. La última sección discute las conclusiones.

\section{CONCEPTOS DE BUSINESS INTELLIGENCE [BI]}

En el entorno empresarial, la tomada de decisiones puede ser considerada una de las etapas esenciales en el proceso de negocio (WU ET AL., 2007). Business Intelligencesurge como un conjunto de tecnologías, técnicas, conceptos y herramientas que auxilian el proceso decisivo y de esta manera permite que las organizaciones optimicen sus recursos de negocio y en consecuencia de esto, mejoren sus resultados.

El principal objetivo de $\mathrm{BI}$ es ofrecer un acceso a los datos de forma simples proporcionando a los administradores de negocio la habilidad de transformar estos datos en informaciones y estas en conocimiento 
de manera que puedan realizar análisis convenientes para tomar decisiones de forma precisa y confiable para el negocio (TURBAN ET AL.,2009).

Para concretizar el proceso de transformación de los datos en informaciones es necesario un ambiente que pueda consolidar y permitir el uso estratégico de los datos extraídos de las bases de datos históricas. Así, estos ambientes almacenan los datos en estructuras dimensionales, permitiendo el procesamiento analítico por medio de herramientas especiales.

Para Turban y otros autores (2009), construir una solución de $\mathrm{BI}$ posee un gran desafío. Actualmente las informaciones están siendo transmitidas en tiempo real, lo que requiere decisiones tomadas con mayor rapidez y con mayor precisión en función de la cantidad de datos disponibles. Esto lleva a la proyección de soluciones de BI para extracción y visualización de esta información.

En este escenario, sistemas de BI proporcionan un ambiente para unificar los datos y ejecutar el proceso de descubierta de conocimiento. Según Turban, otros autores (2009) y Cano (2007), Sistemas de BI poseen cuatro grandes componentes, son ellos:

- Fuentes de Información: Sistemas a través de los cuales son extraídos los datos, tales como, sistemas operacionales y transaccionales de una organización, fuentes de información externa.

- Extracción, Transformación y Carga (ETL): Proceso en el cual los datos son recuperados de los sistemas de origen, permitiendo la depuración por medio de transformaciones para alimentar la base de destino.

- Data Warehouse(DW): Base de datos corporativa que integra datos de diferentes fuentes. Es no volátil y almacena la información de forma organizada preparada en el proceso ETL, para procesarla permitiendo análisis desde múltiples perspectivas.

- Área de presentación: Conjunto de herramientas de BI que permiten la exploración y visualización de la información almacenada en el DW.

La Figura 1 presenta un diagrama que ilustra los componentes de un Sistema BI.

Figura 1 - Componentes de un Sistema de BI

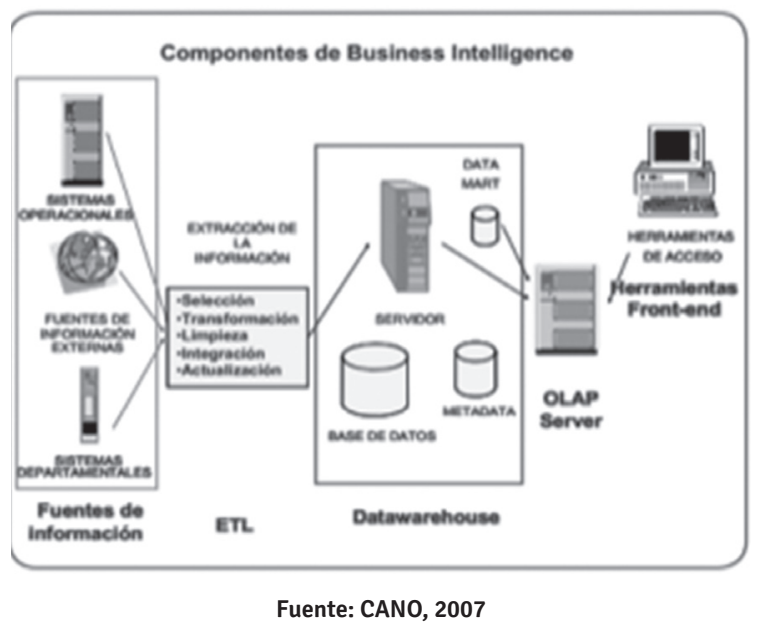

\subsection{DATA MARTS (DM)}

Data Mart pueden ser definidos como una base de datos departamental específica para una determinada área dentro de una organización, enfatizando el fácil acceso a una información relevante. Para Kimball y Ross (2002), Data Marts son subconjuntos de un Data Warehouse completo, con las mismas características.

Para Diaz y Caralt (2012), el objetivo de construir un DM es para responder a un determinado análisis en el interior de una organización. Generalmente estos sistemas almacenan menos información que los DW y permiten acceder a los datos que serán analizados 
de forma rápida pues poseen indexación de almacenamiento. Pudiendo ser tanto dependientes como independientes del DW, construidos con base en arquitecturas Top-Down e Bottom-Up.

\subsection{ON-LINE ANALVTICAL PROCESSING[OLAP]}

Herramientas OLAP son un conjunto de técnicas dirigidas al acceso y análisis ad-hoc de datos, utilizando una serie de recursos para depurar estas informaciones. Se refieren a un conjunto de procesos para integrar, analizar y manipular volúmenes de datos, proporcionando una serie de funcionalidades, objetivando una mayor comprensión de los datos por analistas y administradores del proceso de análisis corporativo.

Diaz y Caralt (2012), explican el concepto como una tecnología que permite posicionar los datos en una visión multidimensional, permitiendo la presentación de las informaciones en distintas perspectivas. La multidimensionalidad es el concepto clave de una herramienta OLAP para sintetizar información, con una visión conceptual personalizada de la información puntual de análisis, o sea, es posible obtener diferentes análisis sobre una misma base de datos posibilitada por el cambio entre las diferentes perspectivas (CANO, 2007).

Este modelo constituye los tipos de datos de negocio y es representado gráficamente por una estructura hipercúbica (CANO, 2007). Es decir, esta estructura permite por medio de varias dimensiones, representar los componentes de negocio disponibles jerárquicamente, permitiendo la visualización de características con el fin de descubrir tendencias y escenarios.

\subsubsection{DESCRIPCIÓN DEL MODELO DE DATOS}

Para modelar los datos de un DW o DM es utilizado el modelo dimensional. Para Kimball y Ross (2002), modelo dimensional es una técnica que posibilita la creación de un modelo de una visión dimen- sional. Este modelo está constituido por un conjunto de medidas que describen los aspectos del negocio. De esta forma es posible agregar y estructurar los datos para dar soporte a los análisis de datos. Tres elementos forman este modelo, son ellos: Dimensiones, Medidas y Hechos.

Las dimensiones son los criterios principales de análisis, contienen las de negocio y se originan a partir de las entidades de las bases de datos de origen. De acuerdo conKimball y Ross (2002), la tabla de hechos esla principal tabla del modelo. Los hechos comprenden los criterios de análisis, son colecciones de ítems de datos compuestas por medidas numéricas que representan la evolución de los negocios de una organización, su llave primaria es una combinación única de los valores de las llaves de sus dimensiones.

\subsubsection{ESQUEMAS DEL MODELO}

Existen dos esquemas principales en un modelo de datos multidimensional, ellos son, StarSchema y Snow Flake(DIAZ y CARALT, 2012). StarSchema consiste en estructurar la información en procesos, vistas y medidas en forma de estrella. En términos de diseño, este esquema está compuesto por una tabla de hechos centralizada, para el hecho analizado, con una o más tablas auxiliares, las tablas de dimensiones, para cada punto de vista de análisis que participa de la descripción del hecho (DIAZ y CARALT, 2012).

El segundo modelo, Snow Flake es un esquema que deriva del modelo StarSchema, donde las tablas de dimensiones se normalizan en diferentes tablas, de esta forma las tabla de hechos deja de ser la única tabla que se relaciona con as otras, y con esto, surgen nuevas uniones (DIAZ y CARALT, 2012).

\subsection{EXTRACT, TRANSFORM AND LOAD [ETL]}

El proceso ETL es de extrema importancia en la construcción de un Sistema de BI, una vez que comprende todos los procedimientos realizados alrededor 
del DW para colectar y transformar los datos previa a la carga del almacén (JUNIOR, 2004). El principal objetivo de este proceso consiste en mantener cargado el almacén con los datos correspondientes.

Este proceso consiste en la utilización de herramientas que realizarán las etapas de colecta y limpieza de los datos de las bases de origen y también la migración de estos datos al DW. Según Júnior (2004) este proceso se presenta en tres etapas, extracción, transformación y carga.

\subsection{SPAGOBI}

Es una plataforma completa que ofrece soporte a los negocios cotidianos y estratégicos tanto a nivel de toma de decisiones como a nivel operacional. El modelo analítico está formado por diversos motores, entre los másutilizados están, los Reports, Indicadores Key Performance Indicator (KPI), Painel de Control, Gráficos, Análisis geográfico e Análisis multidimensional de datos OLAP.

De esta manera, esta herramienta ofrece una solución de $\mathrm{Bl}$ que proporciona soporte al monitoreamiento, análisis y presentación de los datos. Con esto, toda la escalabilidad necesaria en una organización es soportada por SpagoBI, en términos de arquitectura, funcionalidades y seguridad.

\section{METODOLOGÍA}

Conforme Gil (2004), las investigaciones pueden ser clasificadas de acuerdo con su finalidad. La primera clasificación, la investigación básica, objetiva agregar conocimiento por medio de estudios. La segunda, es la investigación aplicada, que engloba estudios a fin de resolver problemas en una situación específica.

Para el autor, las investigaciones, también, pueden ser clasificadas de acuerdo con sus objeti- vos, siendo de carácter exploratorio, descriptivo y explicativo. La investigación exploratoria proporciona un mayor entendimiento sobre el tema abordado y establece criterios, métodos y técnica de planificación flexible para elaborar estudios. Este tipo de proyecto de investigación visa ofrecer informaciones sobre el objeto de estudio yla formulación de hipótesis.

De esta forma, la investigación realizada en este trabajo fue clasificada de forma explicativa y de carácter exploratorio. Con esto, a continuación es descrito el procedimiento adoptado para alcanzar los objetivos propuestos.

\subsection{IMPLEMENTACIÓN DE BI}

La estrategia utilizada para el desenvolvimiento del DM envuelve las principales etapas del modelo propuesto por Kimball y Ross (2002) que se inicia con la planificación del proyecto yla elaboración del entendimiento de las necesidades para implementar BI en UNIPAMPA, así como, de las técnicas y herramientas necesarias para el desenvolvimiento del trabajo.

\subsubsection{DESCRIPCIÓN DE LA ARQUITECTURA}

E neste momento, se hace posible definir la forma con que los componentes del proyecto serán estructurados. Conforme la arquitectura Bottom-Up, la construcción del ambiente DW es realizada a través de la implementación de DM independientes, de esta forma el proyecto es construido de forma evolutiva con retorno rápido y enfoque inicial en los principales negocios (KIMBALL y ROSS, 2002). La Figura 2 ilustra los componentes de la arquitectura de esta aplicación. 
Figura 2 - Arquitectura del Sistema de BI para la aplicación

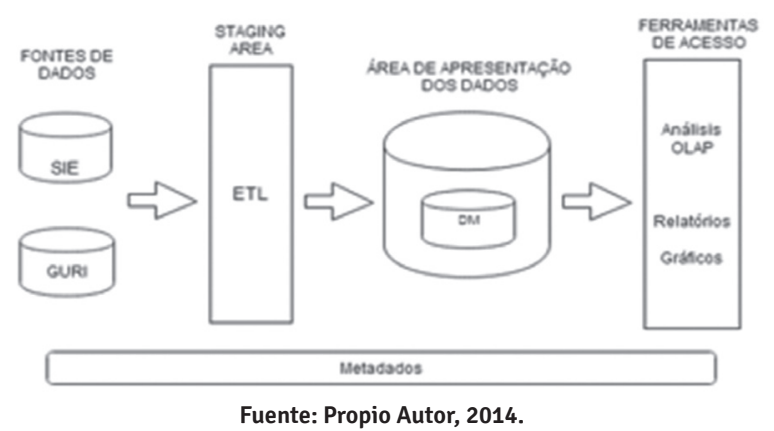

La primera etapa en la creación del DM, consiste en la construcción del modelo dimensional a partir de un sistema transaccional donde es representada la idea central y sus dimensiones, y donde son definidos de qué forma los datos serán almacenados, para permitir consultas de manera rápida y flexible. La etapa siguiente es el proceso ETL, en la cual son realizados los procesos de extracción, transformación y carga de datos de un sistema corporativo para un banco de datos dimensional. Por fin, la visualización de los resultados a partir da herramienta para interacción con el usuario.

La creación del modelo dimensional en proceso de construcción de un DM está compuesta por cuatro pasos: selección del proceso de negocio, definición de granularidad, definición de dimensiones y de hechos (KIMBALL y ROSS, 2002).

\section{Selección del proceso de negocio}

Para la construcción del primero DM para la Institución fue considerado un subconjunto de datos con números de alumnos matriculados, recibidos, ingresados e evadidos da Institución en un determinado período. De esta forma, pueden obtenerse vistas envolviendo cuestiones como, cursos con mayor índice de deserción en relación al número de alumnos cursando y análisis sobre cursos con mayor número de alumnos que concluyen el curso.

\section{Definición de la granularidad}

La definición del nivel granular del registro de la tabla de hechos es un requisito primordial en un proyecto dimensional. Para este proyecto el nivel granular definido para la construcción del DM esta dado en relación a las informaciones designadas por cursos en un periodo. De esta forma, son presentados para cada curso referente a cada campi de la Universidad, las características relacionadas al curso con sus respectivas informaciones por período.

\section{Selección de las tablas de hechos y dimensiones}

En esta etapa fueron definidas y modeladas las tablas necesarias en la implementación del DM. Entre los esquemas de multidimensionalidad estudiados se optó por la utilización del StarSchema, pues propone el desenvolvimiento de proyectos de DM menores y dependientes, fácilmente comprendidos y muy eficientes en la recuperación de datos.

Para el DM propuesto, fueron analizados los requisitos para su creación e identificados los hechos y dimensiones. Con esto, puede ser construido el modelo conceptual identificando los siguientes hechos e visiones dimensionales: Hecho: Inscriptos;Dimensiones: Campus, Cursos, Tempo e Evasão.

Con la definición de las tablas con sus atributos fue construido el modelo físico con auxilio de la herramienta MySQLWorkbench. De esta forma, la Figura 3 representa el diagrama relacional de las estructuras utilizadas para esta aplicación. 
Figura 3 - Modelo Multidimensional en base a tablas relacionales para la aplicación

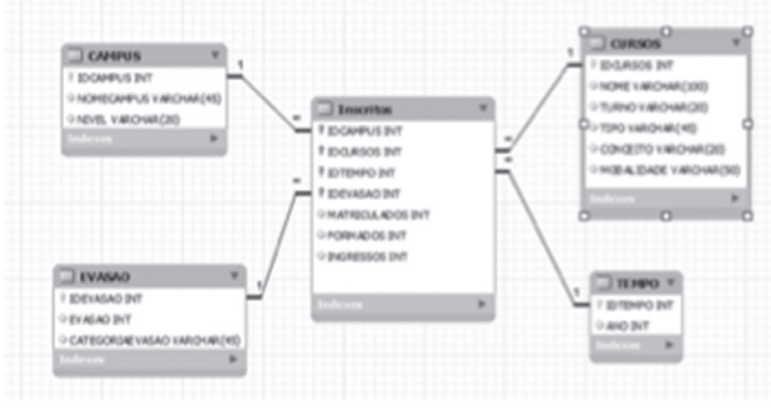

Fuente: Propio Autor, 2014.

El hecho Inscritos modela la cantidad de alumnos inscriptos, ingresados y recibidos en la Universidad según el cruzamiento de las dimensiones: Cursos, Campus, Evasao y Tempo. Cada cantidad es obtenida contando los números de alumnos según un periodo, o sea, durante los años 2012 y 2013.

Una vez realizada la construcción del modelo de datos es posible iniciar e. Así, la subsección siguiente es realizada la descripción este proceso.

\subsubsection{PROCESO ETL}

Para migrar los datos hacia el DM, el primer paso es realizar la extracción de los datos de las fuentes, el principal objetivo de ésta etapa es extraer solamente aquellos datos de los sistemas operacionales que serán necesarios, y prepararlos para las siguientes etapas del proceso ETL (CANO, 2007).

En la etapa siguiente del proceso ETL, los datos son pre-procesados y preparados con el objetivo de mejorar la calidad, generando una base separada para el análisis. Así los datos fueron separados y organizados con las necesidades del proyecto. La limpieza contribuye para eliminar las inconsistencias de la base como: completar datos, tratar valores nulos, y eliminar registros irrelevantes para el análisis.
Para este trabajo, los datos fueron extraídos y preparados con auxilio de la herramienta Talend, para ser cargados para el DM presentando la estructura necesaria para su utilización. En la etapa de la carga fue necesario verificar la integridad entre llaves primarias y secundarias para construir un ambiente analítico íntegro y confiable. De esta manera, se efectuó la carga siguiendo los modelos de la tabla hechos y dimensiones, a partir del script generado por el diagrama del modelo construido anteriormente.

\subsubsection{EXPLORACIÓN DE DATOS}

Para iniciar el proceso de análisis y exploración de datos, fueron realizadas la generación del cubo OLAP, para posibilitar las consultas permitiendo visiones multidimensionales, la creación de gráficos y de reports sobre las consultas.

Para el análisis OLAP los cubos generados a través de SpagoBly construidos a partir de definiciones de las tablas hecho y dimensiones. Con la estructura básica del cubo proyectada y el modelaje dimensional previamente descripta, fueron definidas para cada dimensión los atributos que serían visualizados por usuarios, así como, sus jerarquías. En la etapa siguiente del proceso, fue realizada la visualización de datos tornando las aplicaciones de soporte a decisiones entendibles y permitiendo una mejor interpretación de los datos para posibilitar la identificación de padrones y tendencias.

De esta forma, la técnica OLAP permitió la construcción de gráficos del sistema, posibilitando la visualización de informaciones estructurados a partir de los datos existentes en el sistema. Las operaciones OLAP realizadas incluyen Slice and Dice, visualizando datos sobre diferentes puntos de vista, aplicando diferentes critérios de filtros, Roll Upagregando informaciones detalladas a niveles superiores y Drill Down posibilitando un nivel de detalle de datos más bajos. 


\section{ANÁLISIS DE RESULTADOS}

La Universidad Federal do Pampa, fundada en 2006, es una Institución de Enseñanza Superior multicampi, con su sede localizada en la ciudad de Bagé, Rio Grande do Sul, Brasil. La UNIPAMPA desenvuelve actividades de enseñanza, pesquisa e extensión.

Actualmente, en sus estructuras de almacenamiento, la UNIPAMPA posee más de un millón de registros relatando la vida académica de los estudiantes inscriptos en la Universidad. Estos datos son mantenidos en el Sistema de información corporativo (SIE). Los módulos implantados del SIE incluyen los módulos académicos, de biblioteca, de recursos humanos. En el módulo académico están almacenadas las informaciones relativas a formularios de cursos, de materias, de alumnos con todas sus informaciones relativas a la vida académica de cada estudiante, así como, identificación de alumnos que se reciben en cada curso y el lanzamiento de las notas e inscripciones.

\subsection{APLICACIÓN}

Es posible observar que a pesar de todos los esfuerzos desprendidos en la Institución, la UNIPAMPA aún no dispone recursos efectivos para soporte al proceso de toma de decisiones. Con el fin de limitar el problema y viabilizar la concentración de esfuerzos para conducir a resultados significativos y de su actividad final, fue decidida la implementación de un DM académico.

De esta manera, se pretende viabilizar la utilización de tecnologías para integrar estos datos operacionales de distintas fuentes, de forma consistente y de fácil utilización, permitiendo una mejor gestión administrativa para la Institución. Para demostrar la solución de BI descripta en dicho trabajo, fue utilizado un subconjunto de datos de la Institución. Esta muestra está constituida por 123 registros relativos a cada uno de los cursos con algunos datos de deserción y conclusión.
La finalidad del proceso de análisis de datos es identificar conocimientos nuevos y relevantes. Por otro lado, dado el conjunto de datos restrictos que fue utilizado en este estudio de caso, los análisis apenas presentas padrones estadísticos.

\subsection{CONSULTAS REALIZADAS}

Con la implementación del DM e con auxilio de herramientas de Business Intelligencefue posible construir una base de soporte a la toma de decisiones sobre una de las áreas de interés de la UNIPAMPA. Luego, para los primeros análisis construidos fueron obtenidos y evaluados los resultados.

\section{Inscripciones e Ingresos 2013}

Por medio de análisis OLAP, efectuando operaciones sobre el cubo Slice and Dice, fue posible obtener diferentes vistas sobre los números de inscripciones e ingresantes en cada uno de los cursos. La Figura 4, presenta los datos visualizados.

Figura 4 - Gráfico inscriptos 2012 y 2013

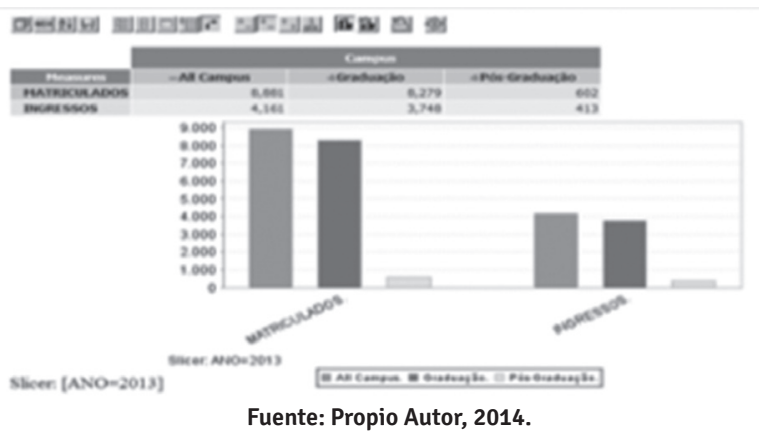

Realizando otras operaciones sobre el cubo, como Drill, fue posible obtener un mayor nivel de detalles, por ejemplo, el número de inscriptos de cada curso de graduación y maestrías correspondientes a cada campi.

\section{Gráfico Alumnos Recibidos por Campi}


El grafico construido, presentado en la Figura 5, ilustra el análisis realizado, donde fue posible observar el porcentaje de alumnos recibidos en un determinado año de un campi especifico.

Figura 5 - Alumnos Recibidos por Campi año 2006

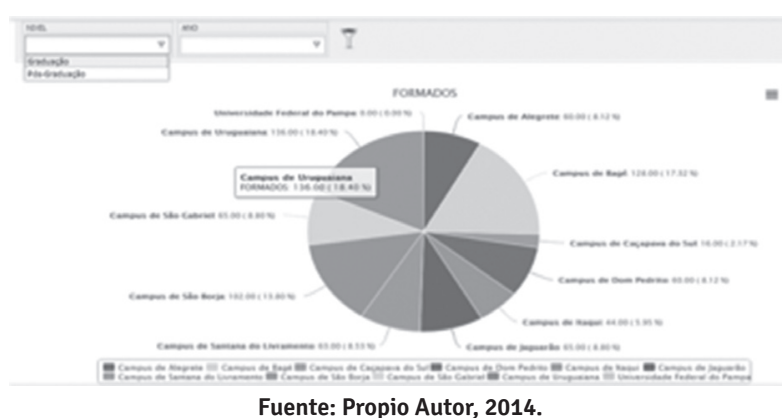

Dashboard

Dashboards proporciona un mecanismo unificador que permite una base gestión eficaz y eficiente para los proyectos. Permiten una presentación de la información más importante, posibilitando al usuario evaluaciones y análisis precisas para alcanzar los objetivos de negocio. La Figura 6, representa un ejemplo de dashboardconstruido en base a los atributos para cada curso.

Figura 6 - Dashboard

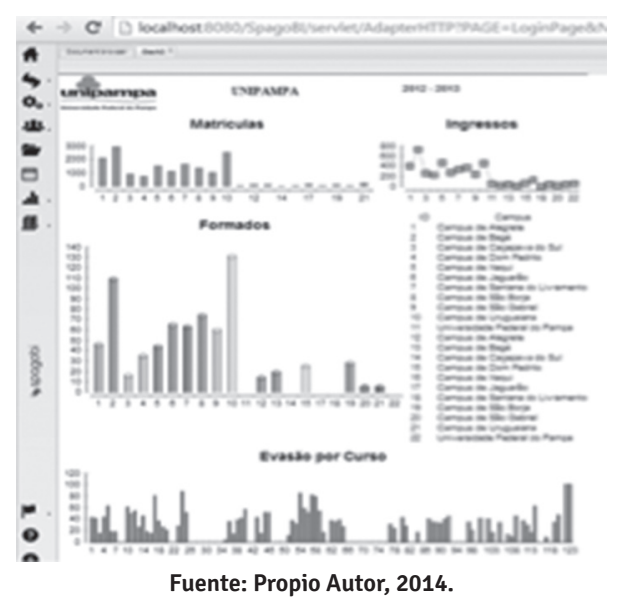

Con los resultados obtenidos, es posible verificar la importancia de implementar BI en la UNIPAMPA. Éste tipo de tecnología posibilita la optimización de la entrega de la información, de forma completa, correcta, consistente, oportuna y accesible.

\section{CONCLUSIONES}

El soporte computacional adecuado y eficiente por parte de los administradores es escaso en la mayoría de las organizaciones. El presente trabajo tuvo como principal objetivo contribuir con la implementación de BI en la Universidad, estableciendo una fuente de información única para el análisis de las actividades realizadas.

Con este trabajo fue posible verificar que la etapa de limpieza, transformación y carga de datos operacionales al DM es una de las etapas más demoradas de éste proceso y la que requiere mayor atención, una vez que errores en las bases operacionales dificultan el proceso ETL y disminuyen la cantidad de datos almacenados en el DM y por consecuencia, los visualizados en el ambiente de Bl.

Además, a través de este trabajo fue posible observar de forma clara los beneficios de la utilización de herramientas de BI. El uso de gráficos y del cubo OLAP posibilitó un mayor dinamismo e interactividad al manipular el DM proporcionando a los tomadores de decisiones análisis de las informaciones precisas.

Finalmente, cabe resaltar la importancia de la construcción de dashboard, en un sistema de Bl. Estos recursos proporcionan un ambiente dinámico e iterativo ofreciendo al usuario la posibilidad de manipular la información de forma clara y objetiva, con esto, pueden ser considerados un importante recurso estratégico para la toma de decisiones.

Las direcciones de investigaciones futuras incluyen: la extracción de nuevos datos de las bases de 
la UNIPAMPA, para mantenerlo actualizado y brindar más informaciones, así como, la construcción de otros dashboards para mostrar otros aspectos del DM con datos académicos.

\section{REFERENCIAS}

CANO, J. L. Business Intelligence: Competir con Información. Barcelona, 2007.

DIAZ, J. C.; CARALT, J. C. Introducción al Bussines Intelligence. Barcelona: UOC, 2012. p.17-28;51-93.

GIL, A. C. Como elaborar projetos de pesquisa. São Paulo: Atlas, 2005. p.26.

INMON, W. H. Como Construir o DataWarehouse. Rio de Janeiro, 1997.

INTERNATIONAL Data Corporation (IDC),Analyzethe future, 2012. Disponible en: <http://www.emc.com/ leadership/digital-universe/iview/index.htm>. Acceso em: 10 de março de 2015
JÚNIOR, M. C. Projetando Sistemas de Apoio à Decisão Baseados em Data Warehouse. 1.ed. Rio de Janeiro: Axcel Books, v.1, 2004.

KIMBALL, R.; Ross, M.The Data Warehouse Toolkit.The Complete Guide to Dimensional Modeling. 2.ed. John Wiley and Sons Inc, 2002.

MySQLWorkbench. Disponible en: <http://www.mysql.com/>. Acceso em:08 de março de 2015.

SPAGOBI. Disponible en: <http://www.spagobi.org/>. Acceso em: 13 de março de 2015

TALEND. Disponible en: <https://www.talend.com>. Acceso em: 13 de março de 2015

TURBAN, E. et al. Business Intelligence: um enfoque gerencial para a inteligência do negócio. Bookman, 2009. WU, L. et. al. A Service-oriented Architecture for Business Intelligence. In: IEEE International Conference on Service-Oriented Computing and Applications(SOCA'07), 2007.

1. Acadêmica do Curso de Engenharia de Computação - Universidade Federal do Pampa (UNIPAMPA. E-mail: anamagelaa@gmail.com

2. Doutor em Computação (UFRGS), Professor Adjunto do Curso Engenharia de Computação - Universidade Federal do Pampa (UNIPAMPA).

E-mail: camargo.sandro@gmail.com 
